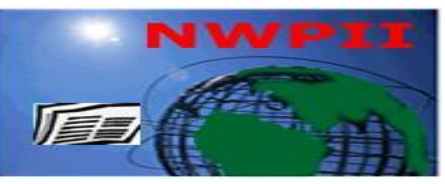

American Journal of Biomedical Sciences

ISSN: 1937-9080

nwpii.com/ajbms

\title{
Is Marijuana Beneficial for Prevention and Treatment of Diabetes?
}

\author{
Rui Cao ${ }^{1,2^{*}}$, Jun Wang ${ }^{1 * \#}$, Wei Zhang ${ }^{1}$, Huang Huang ${ }^{1}$, Yu Qiao ${ }^{3}$, Yufan Dai ${ }^{4}$, \\ Miaozi Gong ${ }^{5}$, Hong C. Lai $^{6}$
}

${ }^{1}$ Department of Pharmacology, Bioengineering and Food College, Hubei University of Technology, Wuhan, Hubei 430068, China

${ }^{2}$ Department of Chemistry, Johns Hopkins University, Baltimore, MD 21218, USA

${ }^{3}$ Research Institute of Agricultural Products Processing and Nuclear-Agricultural Technology, Hubei Academy of Agricultural Sciences, Wuhan 430064, China

${ }^{4}$ School of Physics and Technology, Wuhan University, Wuhan 430072, China

${ }^{5}$ Department of Pathology, Peking University Shougang Hospital, Beijing 100144, China

${ }^{6}$ Department of Radiology, Johns Hopkins School of Medicine, Baltimore, MD 21205, USA

*These two authors contribute equally to this paper.

\#Corresponding Author

Jun Wang

Department of Pharmacology

Bioengineering and Food College

Hubei University of Technology

Wuhan, Hubei 430068

China

Email: 2491549178@qq.com

Received: 02 August 2017; $\mid$ Revised: 05 September 2017; $\mid$ Accepted: 18 December 2017

\section{Abstract}

Marijuana is classified as Cannabis saiva L, one type of hemp. Traditionally, Marijuana is considered as Schedule I drug due to its acute bad effects on human health. However, its medical use has been widely recognized today. Delta-9-tetrahydrocannabinol $\left(\Delta^{9}\right.$-THC) is a compound extracted from Marijuana, which has been used in several drugs approved by United States Food and Drug Administration (FDA). $\Delta^{9}$-THC functions through cannabinoid receptor in neural system or peripheral tissues. For diabetes patients, $\Delta^{9}-\mathrm{THC}$ has been reported to have preventative or treatment effects. In this paper, we will review recent research progress in association between cannabis use and diabetes, as well as known mechanism of how $\Delta^{9}$-THC functions in human from the perspective of free radicals.

Keywords: cannabis,diabetes,Delta-9-tetrahydrocannabinol,free radicals 


\section{Introduction}

Traditionally,federal laws classify marijuana as Schedule I (strictest) drug along with heroine and other hallucinogens by the Controlled Substances Act (1970) due to its high potency for abuse. Marijuana is known to cause a series of acute effects for the users ${ }^{[1]}$ such as anxiety, impaired attention and short-term memory loss, as well as attenuated motor coordination, which pose risks in public settings, such as traffic accident. For longterm marijuana users, the impairment on cognition and memory could be prolonged and irreversible ${ }^{[2]}$. Once heavy marijuana users stopped its intake, they often experience persistent withdrawal symptoms such as nausea, insomnia and depression ${ }^{[2]}$. If not properly regulated, use of marijuana would cause tremendous social crisis and economic loss. Therefore, almost all countries have strict laws regulating use and usage of marijuana.

People often use the name hemp and marijuana interchangeably.The biological classification by the U.S. Department of Agriculture is that hemp is the common name for the genus called "Cannabis L." while marijuana referred to the species "Cannabis sativa L." Moreover, hemp contains only $0.3 \%$ of psychoactive chemicals and mainly planted for its strong fiber that can be used for building materials and textiles. Hemp seeds can also be used to produce hempseed oil which is a legal consumer product in the U.S and research has shown that hempseed oil is rich in essential fatty acids such as omega- 6 and omega- 3 believed by some as "super food" boasting beneficial effects against obesity, diabetes and arthritis. The U.S. imports around $\$ 500$ million worth of hemp product annually from countries such as Canada and China, however, federal laws still prohibit growing of hemp in the U.S. In recent years, states legislatures have taken initiatives to endorse industrial hemp growth, so far 16 states allow industrial hemp growth and 20 states permit research use of hemp growth or pilot program for industrial hemp production. One the other hand, marijuana has about $10 \%$ on average of psychoactive chemicals, which is around 30 times higher than that is found in hemp. In addition, attempts have been made to appeal for reclassification of cannabis including marijuana. Marijuana is now legalized in eight states for adult recreational use; twenty-nine states and Washington, DC imposed laws for allowing medical use of marijuana.

Scientific research on cannabis and its extracted chemicals can be dated as early as $19^{\text {th }}$ century ${ }^{[3]}$. Until 1963, Mechoulam and coworkers ${ }^{[4-6]}$ determined the structure and stereochemistry of the primary psychoactive component of cannabis, i.e. $\Delta^{9}$-tetrahydrocannabinol $\left(\Delta^{9}\right.$-THC), Figure 1a and cannabidiol (CBD), Figure $1 b$.

Zhang and coworkers recently reviewed extraction and analysis of $\Delta^{9}$-THC ${ }^{[7]}$. So far, the FDA approved two synthetic cannabinoid prescription drugs that derived from $\Delta^{9}$-THC, namely Marinol and Cesamet that can be used for treatment of nausea and neuropathic pain after cancer chemotherapy. Chemotherapy drugs, for example, cisplatin induces excessive inflammation and oxidative stress on kidney, renal and neuro system. Meng and coworkers ${ }^{[8]}$ reported a phenolic aldehyde named protocatechuic aldehyde as antioxidant and possible suppress oxidative stress and inflammation poised by cisplatin, potentially operates in a similar fashion as $\Delta^{9}$-THC. Extensive research has been carried out to elucidate the underlying nature for the psychoactive compound $\Delta^{9}$-THC. In 1990, Matsuda and coworkers ${ }^{[9]}$ reported for the first time the THC receptor in mouse brain cells is a G-protein coupled receptor and isolated a complementary DNA sequence that later used to clone the cannabinoid $\left(\Delta^{9}-\mathrm{THC}\right)$ receptor. Gerard and coworkers ${ }^{[10]}$ were able to clone a human cannabinoid receptor using human brain stem cell cDNA. To illuminate the native function of the cannabinoid receptor in the brain, Mechoulam and coworkers [11] isolated and identified the endogenous ligand for the cannabinoid receptor to be anandamide that could act as neurotransmitter for controlling of pleasure. Munro and coworkers ${ }^{[12]}$ reported finding of cannabinoid receptor in the peripheral immune systems such as macrophage cells. The cannabinoid receptors found in the brain as well as central neural systems are often named as CB1 and the ones found in peripheral tissues for controlling of inflammation and immunological reactions are classified as CB2. 


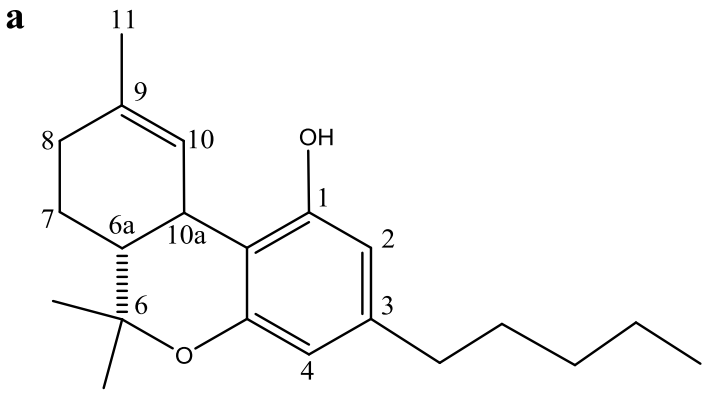

b

Figure 1. a) Chemical Structure of $\Delta^{9}$-THC. b) Chemical Structure of CBD.

\section{Main text}

\subsection{Diabetes as the No.1 health risk}

With the legalization of cannabis and related product, it is necessary to review some of the health consequences for people with regular intake of marijuana. We hereby focus on the recent research data between cannabis product and incidents of diabetes mellitus. Diabetes is becoming the most expensive disease in the U.S., costing over $\$ 100$ billion for diagnosis and treatment per year since $2013^{[13]}$. As of 2014, centers for disease control and prevention (CDC) published statistic data of total diabetes patients in the U.S. to be around 29 million, over 80 million people have prediabetes. Recent advancement in portable personal electronic monitoring device helped with diagnosis of prediabetes ${ }^{[14-16]}$. The total number of diabetes patients in the world was estimated to be around 285 million; the number is projected to be more than 438 million by 2030, Table $1^{[17]}$.

Diabetes mellitus can be classified as type 1 and type 2. Type 1 diabetes arises from auto immune disorders led by destruction of insulin secretary pancreatic beta-cells ${ }^{[17]}$. From a geographical perspective, the prevalence of type 1 diabetes is higher in area with less sunlight such as Finland and lower in area with more sunlight, e.g. India. Furthermore, more cases of type 1 diabetes were diagnosed in winter where sunlight is minimal. This trend is similar to what was found for people with less vitamin $\mathrm{D}_{3}$ synthesis by their skin cells where exposure to sunlight radiation was at reduced amount and higher incidence of cancer, for example
$[18,19]$. From a genetic perspective, certain mutations or disruption of DNA nucleotide base pairs ${ }^{[20]}$, for example, single-nucleotide polymorphism in the gene encoding lymphoid protein tyrosine phosphatase (an enzyme found to attenuate T-cell activation) ${ }^{[21]}$ are associated with type 1 diabetes. The cause of type 2 diabetes is complex and can be attributed to many factors such as high-energy diet and lack of exercise. Wang and coworkers [22] reported inhibition of tyrosine phosphatase enhances insulin sensitivity and could provide therapeutic potential for type 2 diabetes. Cruz and coworkers [23] reported glycine (amino acid normally exists in microhydrated zwitterionic form ${ }^{[24]}$ ) could increase insulin sensitivity and reduce level of glycated hemoglobin.

\subsection{Epidemiological studies that link cannabinoid and decreased diabetes}

Epidemiological studies have linked decreased diabetes incidence with the use of cannabinoid. In 2012, Rajavashisth and coworkers ${ }^{[25]}$ analyzed data collected by the National Health and Nutrition Examination Survey and found that current light marijuana users have lower diabetes prevalence than current heavy users and past marijuana users, Figure 2. Also, all marijuana users (past or current) are less likely to be diagnosed as diabetes compared to non-marijuana users (Figure 2) after adjustment with confronting factors such as age, race and alcohol use. Using logistic regression assessment of the data, the odds ratio (OR) for current light users is 0.29 (95\% CI 0.13 to 0.65 ) compared to non-users. Past marijuana users have odds ratio of 0.44 (95\% CI 0.33 to 0.59 ) and current heavy users have odds ratio of 0.47 (95\% CI 0.22 to 0.98) then non-marijuana users, Figure 2. 
Table 1. Estimated numbers of adults aged 20-79 with any type of diabetes mellitus and prevalence, by region, in 2010 and projected for 2030. ${ }^{[17]}$

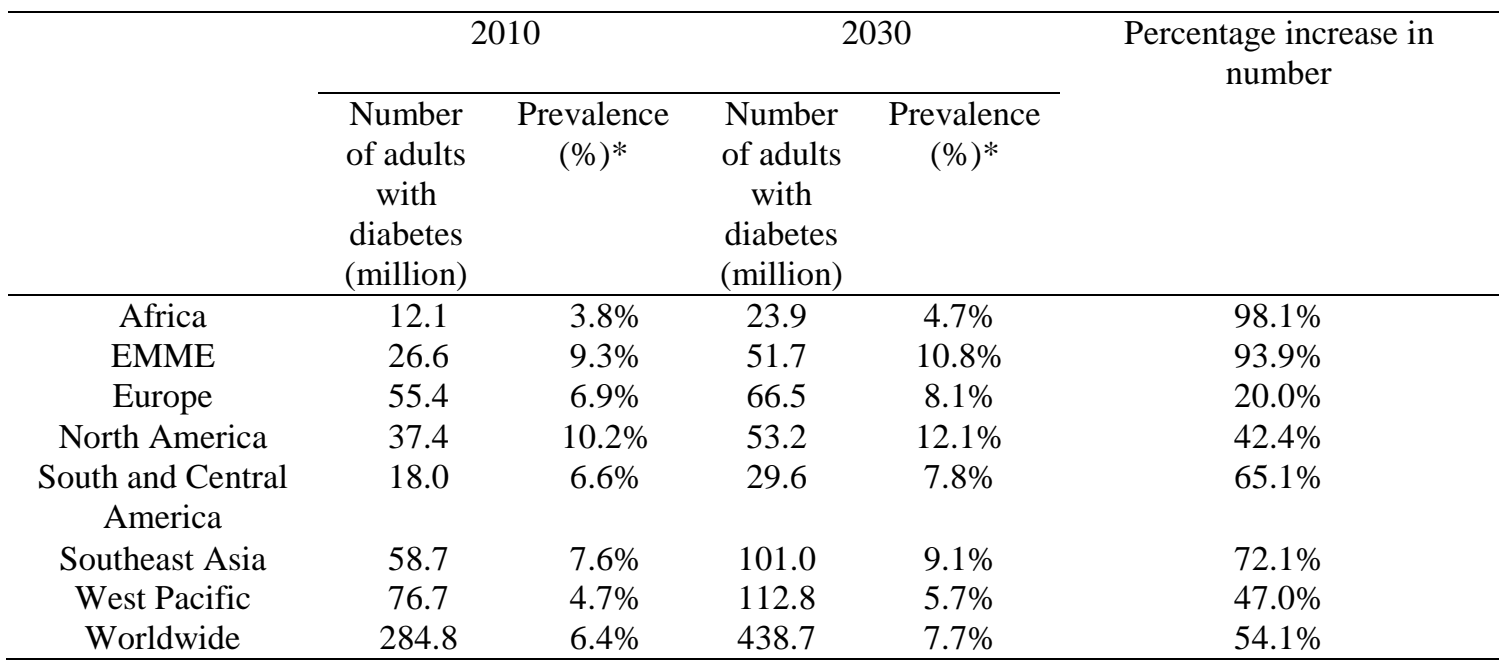

$\mathrm{EMME}=$ Eastern Mediterranean and Middle East. *For each region values are standardized to world age distribution for that year.

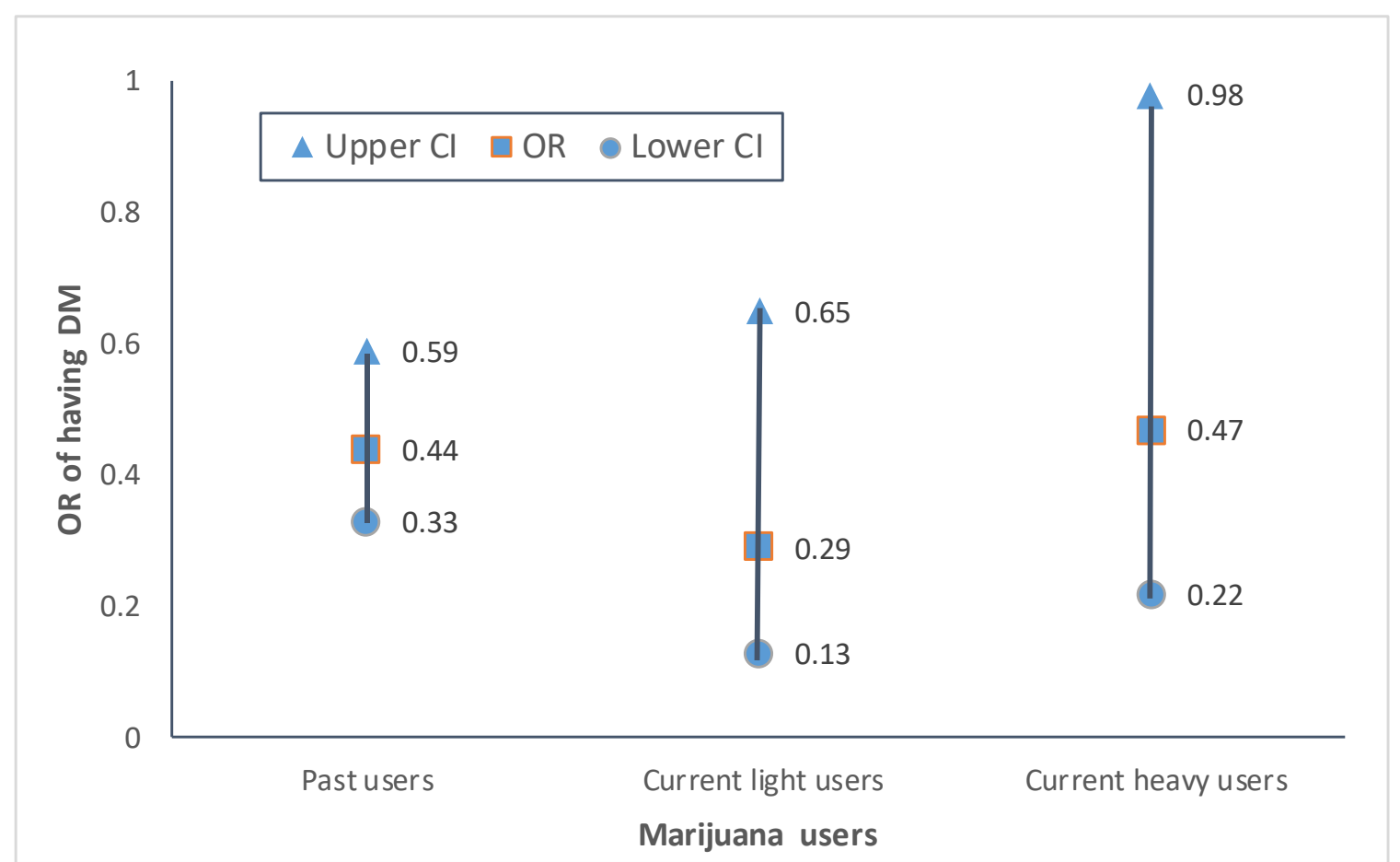

Figure 2. Odds ratio (OR) and 95\% CI of having diabetes mellitus (DM) among past and current marijuana users relative to non-marijuana users.

Later in 2013, Mittleman and coworkers ${ }^{[26]}$ analyzed data from the National Health and Nutrition Examination Survey (NHANES) including 4657 adult men and women from 2005 and 2010. They reported that the correlation between marijuana use and lower fasting insulin level and less insulin resistant is statistically significant, Table 2. The current marijuana users have lower insulin level $(7.5 \mu \mathrm{U} / \mathrm{mL})$ than nonusers $(10.1 \mu \mathrm{U} / \mathrm{ml})$ on average while past users have insulin level between the two groups $(8.8 \mu \mathrm{U} / \mathrm{ml})$. Glucose level is also higher for the non-users $(103.5$ $\mathrm{mg} / \mathrm{dL})$ than current marijuana users $(99.7 \mathrm{mg} / \mathrm{dL})$ and for past users the level is $(100.6 \mathrm{mg} / \mathrm{dL})$. 
Homeostasis model assessment of insulin resistant (HOMA-IR) is again lower for current marijuana users (1.8) when compared to past users (2.2) and non-users (2.5).

The hemoglobin A1c (HbA1c), triglycerides and high-density lipoprotein cholesterol (HDL-C) levels are similar for current, past and non- marijuana users. Body mass index (BMI) is higher for non-marijuana users $\left(29.1 \mathrm{~kg} / \mathrm{m}^{2}\right)$ the number found in past marijuana users $\left(28.5 \mathrm{~kg} / \mathrm{m}^{2}\right)$ as well as current marijuana user $\left(27.2 \mathrm{~kg} / \mathrm{m}^{2}\right)$. Current marijuana users also tend to have smaller waist circumference $(93.6 \mathrm{~cm})$ than past marijuana users $(97.6 \mathrm{~cm})$ and non-marijuana users.

Table 2. Mean values (Standard Errors) of Fasting Insulin and Glucose According to Average Marijuana Use Among Participants from the National Health and Nutrition Examination Survey (NHANES), 2005 to 2010.

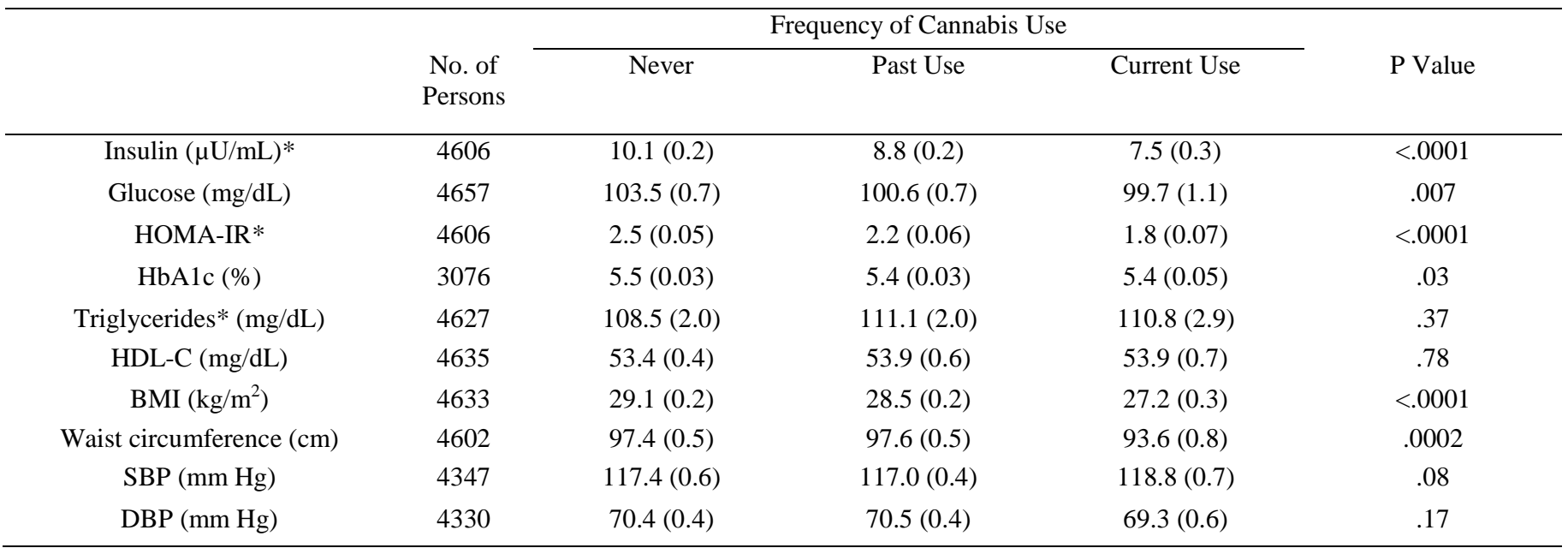

HOMA-IR = homeostasis model assessment of insulin resistance; HbA1c = hemoglobin A1c; HDL-C = high-density lipoprotein cholesterol; BMI = body mass index; $\mathrm{SBP}=$ systolic blood pressure; $\mathrm{DBP}=$ diastolic blood pressure. *Means for insulin, HOMA-IR and triglycerides are geometric.

Anthony and coworkers ${ }^{[27]}$ analyzed data from both National Health and Nutrition Examination Survey (NHANES) as well as the National Surveys on Drug Use and Health (NSDUH) using eight independent sample pools, Table 3 and found that the unadjusted odds ratio of marijuana users to have diabetes mellitus is 0.4 (95\% CI, $0.3-0.5)$ averaging, Table 3. After adjustments for age, sex, ethnics, education, income, alcohol and cigarette smoking, the odds ratio of marijuana users to have diabetes mellitus is 0.7 (95\% CI $0.6-0.8$ ) which is still statistically lower than non-marijuana users to develop diabetes mellitus.

Agardh and coworkers ${ }^{[28]}$ gathered longitudinal data by questionnaires for about 18,000 Swedish men and women and reported marijuana users had lower risk of type 2 diabetes with odds ratio $=0.68$ (95\% CI 0.47 to 0.99 ). In addition, Agardh and coworkers computed the odds ratio fell to 0.94 (95\% CI 0.63 to 1.39 ) after adjustment for age, Table 4. However, in their adjusted odds ratio 0.94 (95\% CI 0.63 to 1.39), they did not differentiate current marijuana users or past marijuana population. In fact, Agardh and coworkers did mention in the paper that the odds ratio is 0.97 for people who used marijuana more than one year ago and the same odds ratio fell to 0.74 when combining the cases in which people used marijuana in the past month or the past year. This result is actually similar to what Anthony and coworkers ${ }^{[28]}$ obtained when they studied data from the National Health and Nutrition Examination Survey (NHANES) and the National Surveys on Drug Use and Health (NSDUH) and obtained the adjusted odds ratio 0.7 (95\% $\quad$ CI 0.6 -0.8). Therefore, based on the lengthened epidemiological studies, marijuana did show at least protective effects again diabetes mellitus. 


\section{The role of Cannabinoid and cannabidiol as antioxidant}

Unhealthy diets usually contain high sugar and energy; the resulting energy surplus can impose great oxidative and nitrosative stress on metabolism. Reactive oxygen species are often generated by molecular oxygen binding to redox active metals such as copper and iron in the human body as side product for energy production. Formation and reactivity of reactive oxygen species are well characterized in ligand copper oxygen model chemistry, for example ${ }^{[29-31]}$. Inoguchi and coworkers ${ }^{[32]}$ reported that high glucose level significantly increases reactive oxygen species production in cultured smooth muscle cells (SMCs) and endothelial cells (ECs) detected by electron spin resonance spectroscopy. Rosen and Lander and coworkers [33] examined the causal effects of reactive oxygen species on the insulin resistant and found that reduced level of reactive oxygen species greatly enhances insulin sensitivity. Insulin resistant is generally considered as the major hallmark of type 2 diabetes. Therefore, reactive oxygen species play a significant role in the development of type 2 diabetes.

Table 3. Study-specific estimates of odds ratios that quantify associations linking occurrence of diabetes mellitus among recently active cannabis smokers: Data for the United States based on eight independent replications from the National Health and Nutrition Examination Survey (NHANES) and the National Surveys on Drug Use and Health (NSDUH), 2005-2012

\begin{tabular}{|c|c|c|c|}
\hline Independent replication sample & $\begin{array}{l}\text { Unadjusted CS-DM } \\
\text { odds ratio }(95 \% \mathrm{CI})\end{array}$ & $\begin{array}{l}\text { Covariate }^{a} \text { adjusted } \\
\text { odds ratio } \\
(95 \% \mathrm{CI})\end{array}$ & $\begin{array}{l}\text { Covariate adjusted odds ratio } \\
\text { additionally adjusted for BMI } \\
(95 \% \mathrm{CI})^{b}\end{array}$ \\
\hline NHANES 2005-06 & $0.3(0.2,0.7)$ & $0.6(0.3,1.2)$ & $0.8(0.4,1.5)$ \\
\hline NSDUH 2005-06 & $0.3(0.2,0.4)$ & $0.7(0.5,1.0)$ & --------------- \\
\hline NHANES 2007-08 & $0.3(0.2,0.5)$ & $0.4(0.2,0.7)$ & $0.4(0.2,0.8)$ \\
\hline NSDUH 2007-08 & $0.3(0.2,0.4)$ & $0.5(0.4,0.7)$ & --------------- \\
\hline NHANES 2009-10 & $0.5(0.3,1.1)$ & $0.9(0.4,2.1)$ & $1.0(0.4,2.1)$ \\
\hline NSDUH 2009-10 & $0.4(0.3,0.6)$ & $0.8(0.5,1.1)$ & --------------- \\
\hline NHANES 2011-12 & $0.5(0.3,0.8)$ & $0.7(0.4,1.2)$ & $0.8(0.4,1.4)$ \\
\hline NSDUH 2011-12 & $0.5(0.4,0.6)$ & $0.9(0.6,1.2)$ & --------------- \\
\hline Meta-analytic odds ratio & $0.4(0.3,0.5)$ & $0.7(0.6,0.8)$ & $0.7(0.5,0.97)$ \\
\hline summary $^{c}$ & & & \\
\hline $\begin{array}{l}\text { Heterogeneity test statistic (p } \\
\text { value) }\end{array}$ & $12.9(0.08)$ & $9.8(0.20)$ & $2.7(0.43)$ \\
\hline
\end{tabular}

${ }^{a}$ Covariate adjustments for age (years), sex (male and female), ethnic self-identification (non-Hispanic Whites, nonHispanic Blacks, Hispanics, and all others), education (less than high school, high school, and above high school), income-poverty ratio $(<1$ and $\geq 1$ ), past-year alcohol drinking (never user, used before but not in the 12 months prior to the interview, and used in the 12 months prior to the interview) and tobacco cigarette smoking (never, former, nondaily, and daily smoker), using the multiple logistic regression model. ${ }^{b}$ Additionally adjusted for BMI $\left(\mathrm{kg} / \mathrm{m}^{2}\right)$. The NSDUH study does not collect data on BMI. ${ }^{c}$ This 'random effects' meta-analysis summary estimate makes an allowance for between-replication variability in the effect estimates. The heterogeneity test statistic (degrees of freedom =7) suggests no appreciable variation (i.e., as gauged in relation to alpha set at 0.05); nonetheless, the 'random effects' summary estimate was retained, with resulting standard errors (and 95\% CI) slightly larger than those obtained using the 'fixed effects' meta-analysis summary estimation approach. 
Table 4. Odds ratios (ORs) and 95\% Confidence Intervals (Cis) for the association between cannabis use in 2002 and subsequent type 2 diabetes (2003 -2011).

\begin{tabular}{|c|c|c|c|}
\hline & \multicolumn{3}{|c|}{ Cannabis use } \\
\hline & \multirow[t]{2}{*}{ Never } & \multicolumn{2}{|c|}{ Ever } \\
\hline & & ORs & (95\% CIs) \\
\hline Crude estimate Adjusted for & 1 & 0.68 & $(0.47-0.99)$ \\
\hline Sex & 1 & 0.65 & $(0.44-0.95)$ \\
\hline Age & 1 & 0.94 & $(0.63-1.39)$ \\
\hline Tobacco smoking & 1 & 0.60 & $(0.41-0.87)$ \\
\hline Physical inactivity & 1 & 0.66 & $(0.46-0.97)$ \\
\hline BMI & 1 & 0.75 & $(0.51-1.10)$ \\
\hline Alcohol use & 1 & 0.67 & $(0.44-1.02)$ \\
\hline Occupational position & 1 & 0.75 & $(0.51-1.09)$ \\
\hline Multiadjusted* & 1 & 0.94 & $(0.63-1.42)$ \\
\hline
\end{tabular}

*Adjusted for age, BMI, tobacco smoking, and occupational position, which changed the OR > 10\%.

Cannabinoids were compared with BHT (butylated hydroxytoluene, a common antioxidant) for their ability to prevent tert-butyl hydroperoxideinduced oxidation of dihydrorhodamine. Data represent mean values SEM from a single experiment with three replicates. This experiment was repeated four times with essentially the same results ${ }^{[34]}$.

The neuroprotective roles of cannabinoid $\left(\Delta^{9}\right.$-THC) and cannabidiol (CBD) have long been reported as antioxidant against reactive oxygen and nitrogen species. Hampson and coworkers [34] reported the protective role of cannabidiol and cannabinoid against hydrogen peroxide ${ }^{[34]}$ where tert-butyl hydroperoxide-induced toxicity was examined in the presence of cannabidiol. The y-axis represents lactate dehydrogenase (LDH) released into the media by dying cells in percentage against control group. Cannabidiol $\left(\Delta^{9}-\mathrm{THC}\right)$ exhibits half maximal effective concentration $\left(\mathrm{EC}_{50}\right)$ to be $6.2 \pm$ $0.1 \mu \mathrm{M}$ and the maximum protective effect again hydrogen peroxide is around $90 \%$. In a separate experiment, Hampson and coworkers ${ }^{[34]}$ tested cannabinoid $\left(\Delta^{9}\right.$-THC), cannabidiol (CBD) and butylated hydroxytoluene (BHT, a common antioxidant) for their ability to prevent tert-butyl hydroperoxide-induced oxidation of dihydrorhodamine, which is a fluorescent reagent for detection of reactive oxygen species [34]. Cannabinoid $\left(\Delta^{9}-\mathrm{THC}\right)$ is shown as effective as NHT with half maximal effective concentration
$\left(\mathrm{EC}_{50}\right)$ to be $18 \pm 1 \mu \mathrm{M}$ which cannabidiol (CBD) is more effective with half maximal effective concentration $\left(\mathrm{EC}_{50}\right)$ to be $11 \pm 1 \mu \mathrm{M}^{[34]}$. In addition, both cannabinoid $\left(\Delta^{9}-\mathrm{THC}\right)$ and cannabidiol (CBD) have shown protective roles against toxic amount of glutamate, which is an important neurotransmitter ${ }^{[34]}$. Downregulation mechanism for cannabinoid $\left(\Delta^{9}-\right.$ THC $)$ and cannabidiol (CBD) have been proposed by Jourdan and coworkers ${ }^{[35]}$ on the cannabinoid receptors. This means rather than activation of CB1 receptor, cannabinoid $\left(\Delta^{9}\right.$-THC) was shown to desensitize the cannabinoid receptors and exhibited protective effects of chronic cannabinoid $\left(\Delta^{9}\right.$-THC $)$ administration.

\section{Anti-inflammatory role of cannabidiol}

Type 1 diabetes is an autoimmune disorder disease led by immune-associated destruction of insulin-producing pancreatic $\beta$ cells ${ }^{[17]}$. Immune system can be activated by inducible nitric oxide synthase (iNOS) ${ }^{[36]}$ upon invading pathogens or $\mathrm{NO}_{\mathrm{x}}$ pollution in the atmosphere ${ }^{[37]}$. Nitric oxide is also a signaling agent and it can be detected via a number of spectroscopic techniques ${ }^{[38]}$. However, excessive nitric oxide production can lead to tissue damaging effects via formation of reactive nitrogen species. In the case of inflammation and immune reactions, reactive nitrogen species are generated and could result in post-translational modification 
on protein, lipids and DNAs. One example is shown as tyrosine nitration imposed by iron or copper peroxynitrite complex $[31,39,40]$. Weiss and coworkers reported ${ }^{[41]}$ that treating type 1 diabetes prone mice with $5 \mathrm{mg} / \mathrm{kg}$ of cannabidiol (CBD) significantly reduce occurrences of type 1 diabetes, compared to vehicle control and untreated mice, Figure 3.

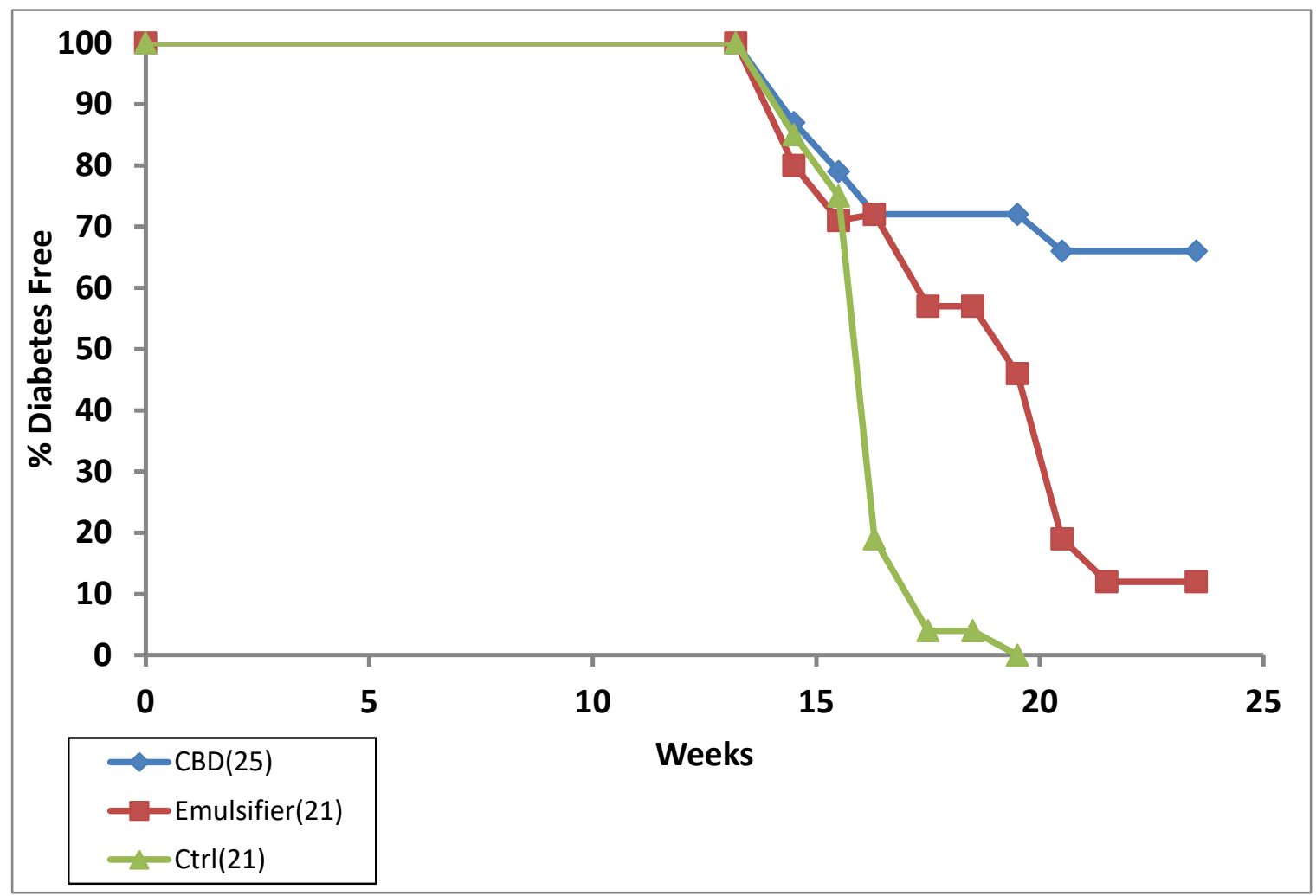

Figure 3. Incidence of diabetes in NOD mice treated with $5 \mathrm{mg} / \mathrm{kg}$ of CBD, vehicle control and untreated mice. The incidence of diabetes in the CBD treated group, compared with vehicle-treated mice and untreated controls was significant $\mathrm{p}<0.001$ (log tank test) ${ }^{[41]}$

After about 21 weeks, untreated mice all developed diabetes while over $80 \%$ of vehicletreated mice have also developed diabetes, Figure 3. With CBD treated group, the incidence of diabetes mice plateaued at 18 weeks and only $30 \%$ mice developed diabetes through 25 weeks, Figure 3. Since cannabidiol is neither agonist or antagonist for the cannabinoid receptor, a cannabinoid receptor-independent mechanism was proposed for the protective effects of cannabidiol ${ }^{[35]}$.

\section{Summary}

Recent research of cannabis products has shed light on diabetes diseases, though researchers and pharmaceutical companies are still cautious due to strict federal law regulation and traditional opinion of these products. Decreased prevalence of diabetes was found in current light marijuana users, compared to current heavy users and past users. Lower fasting insulin level and less insulin resistance was correlated with marijuana use. To pursue the mechanism of how marijuana protects human from diabetes, Delta-9-tetrahydrocannabinol $\left(\Delta^{9}\right.$-THC) and cannabidiol were brought into researchers' attention. Reduced reactive oxygen species are found to greatly enhance insulin sensitivity. Insulin resistance is a major hallmark of type 2 diabetes. $\Delta^{9}$-THC has been repeatedly reported as antioxidant against reactive oxygen species and nitrogen species, such as hydrogen peroxide. $\Delta^{9}$-THC has shown capability to 
desensitize the cannabinoid receptor and exhibited protective effects of chronic cannabinoid administration. The diabetic syndromes were significantly relieved in diabetic type 1 mice after treatment of cannabidiol. All these evidences have shown promising medical effect of THC and cannabidiol. Further work may focus on further elucidation of mechanisms, toxicity of different doses and clinical trials.

\section{References}

1. Hall W, Solowij N. Adverse effects of cannabis. Lancet. $\quad$ 1998;352(9140):1611-6. doi: 10.1016/S0140-6736(98)05021-1.

2. Hall W, Degenhardt L. Adverse health effects of non-medical cannabis use. Lancet. 2009;374(9698):1383-91. doi: 10.1016/S01406736(09)61037-0.

3. Mechoulam R, Hanus L. A historical overview of chemical research on cannabinoids. Chem Phys Lipids. 2000;108(1-2):1-13. doi: 10.1016/S0009-3084(00)00184-5.

4. Michoulam R, Shvo Y. Hashish. I. The structure of cannabidiol.

Tetrahedron. 1963;19(12):2073-8.

5. Gaoni Y, Mechoulam R. Isolation, Structure, and Partial Synthesis of an Active Constituent of Hashish. J Am Chem Soc. 1964;86(8):1646. doi: 10.1021/ja01062a046.

6. Mechoulam R, Gaoni Y. Absolute Configuration of Delta1-Tetrahydrocannabinol Major Active Constituent of Hashish. Tetrahedron Lett. 1967;(12):1109

7. Zhang W, Wang J, Mi Z, Su J, You X, Keceli $\mathrm{G}$, et al. Extraction and Analysis of Tetrahydrocannabinol, A Cannabis Compound in Oral Fluid. International Journal of Biology. 2016;9(1):30.

8. Gao L, Wu WF, Dong L, Ren GL, Li HD, Yang $\mathrm{Q}$, et al. Protocatechuic Aldehyde Attenuates Cisplatin-Induced Acute Kidney Injury by Suppressing Nox-Mediated Oxidative Stress and Renal Inflammation. Front Pharmacol. 2016;7:479. doi: 10.3389/fphar.2016.00479.

9. Matsuda LA, Lolait SJ, Brownstein MJ, Young AC, Bonner TI. Structure of a Cannabinoid Receptor and Functional Expression of the Cloned Cdna. Nature. 1990;346(6284):561-4.
10. Gerard CM, Mollereau C, Vassart G, Parmentier M. Molecular-Cloning of a Human Cannabinoid Receptor Which Is Also Expressed in Testis. Biochem $\boldsymbol{J}$. 1991;279:129-34.

11. Devane WA, Hanus L, Breuer A, Pertwee RG, Stevenson LA, Griffin G, et al. Isolation and Structure of a Brain Constituent That Binds to the Cannabinoid Receptor.Science. 1992;258(5090):1946-9.doi: 10.1126/science.1470919.

12. Munro S, Thomas KL, Abu-Shaar M. Molecular characterization of a peripheral receptor for cannabinoids. Nature. 1993;365(6441):61-5. doi: 10.1038/365061a0. PMID: 7689702.

13. Dieleman JL, Baral R, Birger M, Bui AL, Bulchis A, Chapin A, et al. US Spending on Personal Health Care and Public Health, 19962013. JAMA. 2016;316(24):2627-46. doi: 10.1001/jama.2016.16885.

14. Cai W, Huang L, Wang S. Class D Power Amplifier for Medical Application. Informatics Engineering, an International Journal. 2016;4(2):09-15. doi: 10.5121/ieij.2016.4202.

15. Cai W, Huang L, Wen W. Low Power Class AB SI Power Amplifier for Wireless Medical Sensor Network. Bioscience \& Engineering : An International Journal. 2016;3(3):01-8. doi: 10.5121/bioej.2016.3301.

16. Cai W, Li C, Gu H. Low Power Si-Based Power Amplifier for Healthcare Application. International Journal of Pharmacy and Pharmaceutical Sciences. 2016;8(9):307. doi: 10.22159/ijpps.2016v8i9.12141.

17. Nolan CJ, Damm P, Prentki M. Type 2 diabetes across generations: from pathophysiology to prevention and management. Lancet. 2011;378(9786):169-81. doi: 10.1016/S01406736(11)60614-4.

18. Wang J, Cao R, Zhang W, Mi Z, Su J, You X, et al. Whether Vitamin D Can Prevent Cancer or Not: Recent Research Progress in Vitamin $\mathrm{D}$ and Major Cancers. International Journal of Current Research in Biosciences and Plant Biology.2016;3(11):108-14.doi: 10.20546/ijcrbp.2016.311.015.

19. Zhang W, Wang J, Cao R, Lai HC, Mi Z, Su J, et al. Vitamin D Assay in Human Serum 
Samples: A Review of Analysis Methods. International Journal of Current Research in Biosciences and Plant Biology. 2017;4(2):1-6. doi: 10.20546/ijcrbp.2017.402.001.

20. Ko YJ, Wang HP, Cao R, Radisic D, Eustis SN, Stokes ST, et al. Photoelectron spectroscopy of homogeneous nucleic acid base dimer anions. Phys Chem Chem Phys. 2010;12(14):3535-41. doi: 10.1039/b924950h.

21. Bottini N, Musumeci L, Alonso A, Rahmouni S, Nika K, Rostamkhani M, et al. A functional variant of lymphoid tyrosine phosphatase is associated with type I diabetes. Nat Genet. 2004;36(4):337-8. doi: 10.1038/ng1323.

22. Wang M, Li X, Dong L, Chen X, Xu W, Wang R. Virtual screening, optimization, and identification of a novel specific PTP-MEG2 Inhibitor with potential therapy for T2DM. Oncotarget. 2016;7(32):50828-34. doi: 10.18632/oncotarget.10341.

23. Cruz M, Maldonado-Bernal C, MondragonGonzalez R, Sanchez-Barrera R, Wacher NH, Carvajal-Sandoval G, et al. Glycine treatment decreases proinflammatory cytokines and increases interferon-gamma in patients with type 2 diabetes. J Endocrinol Invest. 2008;31(8):694-9.

24. Tian SX, Sun X, Cao R, Yang JL. Thermal Stabilities of the Microhydrated Zwitterionic Glycine: A Kinetics and Dynamics Study. J Phys Chem A. 2009;113(2):480-3. doi: 10.1021/jp8092594.

25. Rajavashisth TB, Shaheen M, Norris KC, Pan D, Sinha SK, Ortega J, et al. Decreased prevalence of diabetes in marijuana users: cross-sectional data from the National Health and Nutrition Examination Survey (NHANES) III. BMJ Open. 2012(2):e000494. doi: 10.1136/bmjopen-2011-000494.

26. Penner EA, Buettner H, Mittleman MA. The Impact of Marijuana Use on Glucose, Insulin, and Insulin Resistance among US Adults. Am J Med. 2013;126(7):583-9. doi: 10.1016/j.amjmed.2013.03.002.

27. Alshaarawy O, Anthony JC. Cannabis Smoking and Diabetes Mellitus: Results from Metaanalysis with Eight Independent Replication Samples. Epidemiology. 2015;26(4):597-600. doi: 10.1097/EDE.0000000000000314.
28. Danielsson AK, Lundin A, Yaregal A, Otstenson CG, Allebeck P, Agardh EE. Cannabis Use as Risk or Protection for Type 2 Diabetes: A Longitudinal Study of 18000 Swedish Men and Women. J Diabetes Res. 2016.doi:Artn 6278709/10.1155/2016/6278709.

29. Karlin KD, Peterson RL, Kim S, Lee JY, Cao R. Ligand effects on the formation, structure, and reactivity of primary copper-dioxygen adducts. Abstr Pap Am Chem S. 2014;248.

30. Cao R, Saracini C, Ginsbach JW, KieberEmmons MT, Siegler MA, Solomon EI, et al. Peroxo and Superoxo Moieties Bound to Copper Ion: Electron-Transfer Equilibrium with a Small Reorganization Energy. $\boldsymbol{J}$ Am Chem Soc. 2016;138(22):7055-66. doi: 10.1021/jacs.6b02404.

31. Cao R. Binuclear Ligand Copper Dioxygen.Nitric Oxide Adducts: Characterization and Reactivity: Johns Hopkins University; 2016.

32. Inoguchi T, Li P, Umeda F, Yu HY, Kakimoto M, Imamura $\mathrm{M}$, et al. High glucose level and free fatty acid stimulate reactive oxygen species production through protein kinase $\mathrm{C}$ dependent activation of $\mathrm{NAD}(\mathrm{P}) \mathrm{H}$ oxidase in cultured vascular cells. Diabetes. 2000;49(11):1939-45. doi: $\underline{10.2337 / \text { diabetes.49.11.1939. }}$

33. Houstis N, Rosen ED, Lander ES. Reactive oxygen species have a causal role in multiple forms of insulin resistance. Nature. 2006;440(7086):944-8. doi: 10.1038/nature04634.

34. Hampson AJ, Grimaldi M, Axelrod J, Wink D. Cannabidiol and (-)Delta(9)tetrahydrocannabinol are neuroprotective antioxidants. P Natl Acad Sci USA. 1998;95(14):8268-73. doi: 10.1073/pnas.95.14.8268.

35. Jourdan T, Godlewski G, Kunos G. Endocannabinoid regulation of beta-cell functions: implications for glycaemic control and diabetes. Diabetes Obes Metab. 2016;18(6):549-57. doi: 10.1111/dom.12646.

36. Bogdan C. Nitric oxide and the immune response. Nat Immunol. 2001;2(10):907-16. doi: 10.1038/ni1001-907.

37. Wang J, Zhang W, Cao R, You X, Lai H. 
Analysis of Nitrogen Dioxide in Environment. Advances in Bioscience and Biotechnology. 2016;07(06):278-88. doi: 10.4236/abb.2016.76026.

38. Wang J, Keceli G, Cao R, Su J, Mi Z. Molybdenum-containing nitrite reductases: Spectroscopic characterization and redox mechanism. Redox Rep. 2017;22(1):17-25. doi: 10.1080/13510002.2016.1206175.

39. Karlin KD, Sharma SK, Cao R. Peroxynitrite $(\mathrm{O}=\mathrm{NOO}-)$ generation at metal complex centers via (ligand)-M/NO/O-2 reactivity. Abstr Pap Am Chem S. 2014;248.
40. Cao R, Elrod LT, Lehane RL, Kim E, Karlin KD. A Peroxynitrite Dicopper Complex: Formation via $\mathrm{Cu}-\mathrm{NO}$ and $\mathrm{Cu}-\mathrm{O} 2$ Intermediates and Reactivity via O-O Cleavage Chemistry.J Am Chem Soc. 2016;138(49):16148-58. doi: $10.1021 /$ jacs.6b10689.

41. Weiss L, Zeira M, Reich S, Slavin S, Raz I, Mechoulam R, et al. Cannabidiol arrests onset of autoimmune diabetes in NOD mice. Neuropharmacology. 2008;54(1):244-9. doi: 10.1016/j.neuropharm.2007.06.029. 